\title{
Iron Based Magnetoreception in the Honey Bee Apis mellifera
}

\author{
Alastair Boyd ${ }^{1}$, Michael House ${ }^{2}$, Gary Cowin $^{3}$, Boris Baer ${ }^{4}$, Peta Clode ${ }^{1}$, Jeremy Shaw ${ }^{1}$ \\ ${ }^{1 .}$ Centre for Microscopy, Characterisation and Analysis, The University of Western \\ Australia, Perth, Australia. \\ 2. School of Physics, The University of Western Australia, Perth, Australia. \\ 3. Centre for Advanced Imaging, The University of Queensland, Brisbane, Australia. \\ 4. Centre for Integrative Bee Research, The University of Western Australia, Perth, Australia.
}

It is widely accepted that many organisms are capable of detecting and responding to the Earth's magnetic field. This sense has been demonstrated behaviourally and physiologically in animals undertaking a range of activities including long distance migration and a variety of other orientation behaviours. Despite these observations, the cellular mechanisms responsible for magnetoreception remain elusive.

One of the foremost theories relating to this sensory method is based on very small displacements of nanoscale iron-oxide crystals attached to cellular membranes. A primitive version of this mechanism has been well characterized in magnetotactic bacteria. These possess chains comprised of $\sim 100 \mathrm{~nm}$ sized magnetite crystals $\left(\mathrm{Fe}_{3} \mathrm{O}_{4}\right)$ (Figure 1), which aid in orienting the swimming direction of these bacteria towards optimum living conditions. A related magnetoreceptor in higher order species has yet to be definitively identified and characterised.

The worker caste of the honey bee Apis mellifera is an ideal model system for studying magnetoreception as they possess well developed cognitive functions [1], including magnetoreceptive behaviour [2]. Honey bees have been shown to respond to external magnetic stimuli when communicating, foraging, comb building and, most notably, when reference points such as the sun or gravity cues are absent [3]. Other experimental advantages include the animal's social structure and large numbers of small sized individuals.

To locate the magnetic sensory mechanisms within the honey bee our approach involves a combination of analytical techniques ranging from bulk methods involving large sample sizes to detailed microscopic observations, previously untried in this field.

Inductively coupled plasma atomic emission spectroscopy (ICP-AES) and superconducting quantum interference device magnetometry (SQUID) allow for large volumes of tissue to be analysed for traces of magnetic iron. While these methods are limited to assessing relatively large body parts, areas of interest can be indentified for further analysis using higher resolution imaging techniques.

Magnetic resonance imaging (MRI) is sensitive to the changes in magnetic susceptibility across both specific parts of a bee as well as an entire specimen. Different scan types can be used to highlight structural or magnetically influenced aspects of the specimen (Figure 2), allowing for targeting of specific features. Although bulk studies have revealed the crystals are $100 \mathrm{~nm}$ in size and less, the highly magnetic nature of magnetite can allow even cellular volumes to appear visible [4]. 
By employing light microscopy stains in conjunction with serial sectioning, collections of iron containing cells can be located amongst large volumes of tissue (Figure 3). When areas of interest are identified, electron microscopy can then be employed to characterise the structure and composition of the candidate magnetoreceptor cells. Cryo-preparation of biological tissues will ensure that the structural detail needed to describe cell function is retained. Elemental analyses can then allow for both the identification and characterisation of the iron-oxide deposits in the appropriate cells (Figure 4). Through this systematic approach of low to high resolution analyses, we can begin to understand the complex cellular mechanisms involved in magnetoreception and reveal the intricacies of the magnetic sense.

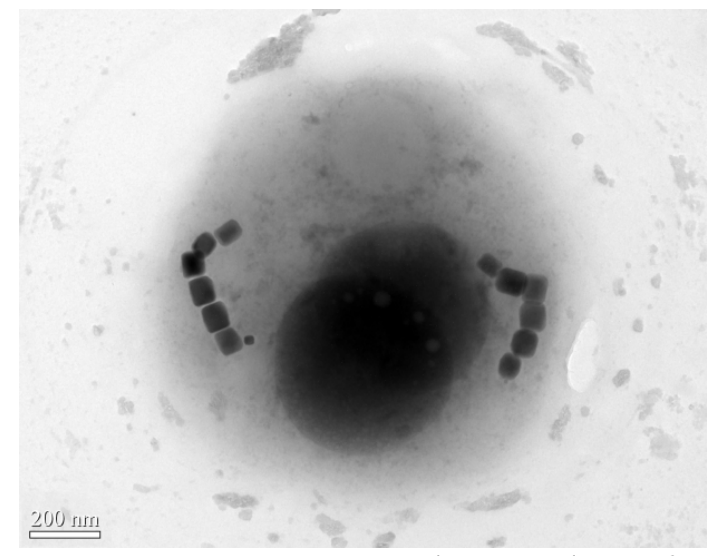

Figure 1. TEM micrograph of a magnetotactic bacterium, demonstrating the presence of nanoscale magnetite chains.

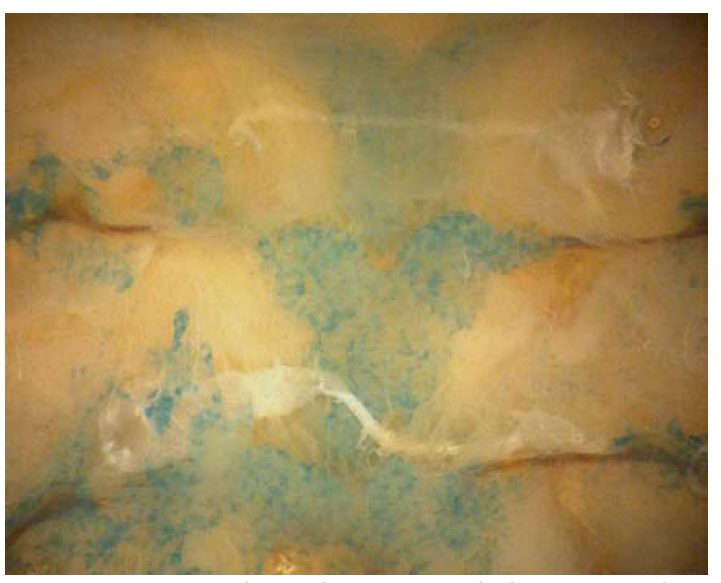

Figure 3. Splayed open abdomen of an adult honey bee stained with Peals Prussian Blue. The highlighting of cells within the fat layer indicates the presence of iron.

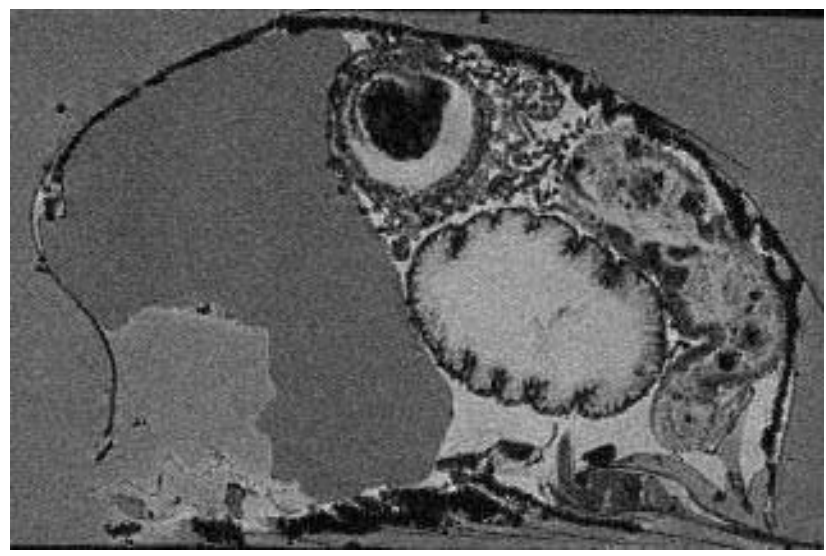

Figure 2. MRI slice portraying the internal structure of a honey bee abdomen. Iron loaded fat cells can be seen as dark material below the cuticle, particularly along the ventral side of the abdomen.

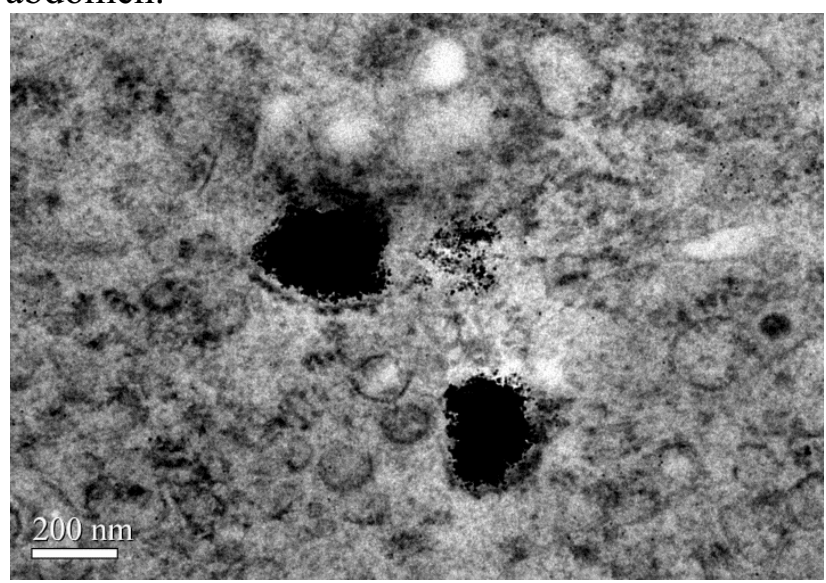

Figure 4. TEM micrograph of iron containing granules found within honey bee trophocytes.

\section{References}

[1] Menzel R, Giurfa M., Behavioral and Cognitive Neuroscience Reviews 5 (2006), p. 2440.

[2] Kirschvink, J. L., Kirschvink, A. K., American Zoologist 31 (1991), p. 169-185.

[3] Wajnberg E et al, Journal of the Royal Society Interface 7 (2010), p. S207-S225.

[4] Foster-Gareau P et al, Magnetic Resonance in Medicine 49 (2003), p. 968-971. 